\title{
OPTIMAL CONTROL OF THE LINEARIZED NAVIER-STOKES SYSTEM IN A NETLIKE DOMAIN
}

1 Voronezh State University, 1, Universitetskaya square, Voronezh, 394006, Russian Federation

2 Voronezh State Technical University, 14, Moskovskii pr., Voronezh, 394026, Russian Federation

This work is a natural extension of research into optimal control problems of evolution equations with distributed parameters on a geometrical graph (network) of one of the authors in the direction of increasing the dimension of a spatial variable and the functions describing the state of the study of the Navier-Stokes equations. At the same time is examined a simple case of the absence of convective effect (laminar flow of an incompressible viscous fluid) linearized system of Navier-Stokes equations in a net-like domain. It proves unique solvability of the initial boundary value problem in the weak formulation which is based on the FaedoGalerkin method using a special basis (the set of generalized eigenfunctions of the special spectral problem) and a priori estimates of norms solutions such as power inequalities. The proof is constructive: to construct a sequence of approximate solutions that converges weakly to the exact solution of the problem. Problems are analyzed with distributed and a start control with a final observation, widespread in applications, that provides the necessary and sufficient conditions for the existence of optimal controls in terms of the conjugate states of the respective systems. Sufficient attention is paid to the synthesis of the optimal control action, and analogues of established finite-dimensional case for Kalman results have been obtained. Although, the use of this method is demonstrated by examples of optimal control theory, this method has a highly susceptible to generalization and applicable to a wide class of linear problems. Refs 15 .

Keywords: linearized Navier-Stokes system, net like domain, weak solutions, optimal control, control synthesis.

\section{B. В. Провоторов ${ }^{1}$, Е. Н. Провоторова ${ }^{2}$}

\section{ОПТИМАЛЬНОЕ УПРАВЛЕНИЕ ЛИНЕАРИЗОВАННОЙ СИСТЕМОЙ НАВЬЕ-СТОКСА В СЕТЕПОДОБНОЙ ОБЛАСТИ}

1 Воронежский государственный университет, Российская Федерация, 394006, Воронеж, Университетская площадь, 1

2 Воронежский государственный технический университет, Российская Федерация, 394026, Воронеж, Московский пр., 14

Рассматривается начально-краевая задача для линеаризованной системы Навье-Стокса в сетеподобной области, в основу анализа которой положены результаты О. А. Ладыженской, Н. Н. Уральцевой, Ж.-Л. Лионса и А. В. Фурсикова для уравнений с частными производными в классических областях. Методом Фаэдо-Галеркина, используя специальный базис (множество обобщенных собственных функций специальной спектральной задачи)

Provotorov Vyacheslav Vasil'evich - doctor of physics and mathematics sciences, professor; wwprov@mail.ru

Provotorova Elena Nikolaevna - PhD of physical and mathematical sciences, associate professor; enprov@mail.ru

Провоторов Вячеслав Василъевич - доктор физико-математических наук, профессор; wwprov@mail.ru

Провоторова Елена Николаевна - кандидат физико-математических наук, доцент; enprov@mail.ru

(C) Санкт-Петербургский государственный университет, 2017 
и априорные оценки нормы решения типа энергетических неравенств, показана однозначная разрешимость рассматриваемой начально-краевой задачи в слабой постановке. При этом строится последовательность приближенных решений, слабо сходящаяся к точному решению задачи. Приведен анализ распространенных в приложениях задач распределенного и стартового управлений с финальным наблюдением, получены необходимые и достаточные условия существования оптимальных управлений в терминах сопряженных состояний соответствующих систем. Решена задача синтеза оптимального управления для случая отсутствия ограничений на управляющие воздействия и получены аналоги известных для конечномерного случая результатов Калмана. Используемый в работе подход является естественным при анализе широкого класса задач оптимального управления, которые описываются с помощью корректных по Адамару начально-краевых задач; представленные результаты являются основополагающими при исследовании задач оптимального управления динамикой ламинарных течений многофазных сред, а также при изучении поведения плоских и сферических волн с сильным разрывом на фронте, который двигается с заданной скоростью. Библиогр. 15 назв.

Ключевые слова: линеаризованная система Навье-Стокса, сетеподобная область, слабые решения, оптимальное управление, синтез управления.

1. Introduction. In the applied problems of hydrodynamics, the system of two equations

$$
\begin{gathered}
\frac{\partial Y}{\partial t}-\nu \triangle Y+\sum_{i=1}^{n} Y_{i} \frac{\partial Y}{\partial x_{i}}+\nabla p=f, \\
\operatorname{div} Y=0\left(\sum_{i=1}^{n} \frac{\partial Y}{\partial x_{i}}=0\right)
\end{gathered}
$$

in a pair of functions $\{Y(x, t), p(x, t)\}$ (the Navier-Stokes system in the evolutionary case [1, p. 77], where $Y=\left\{Y_{1}, Y_{2}, \ldots, Y_{n}\right\}$ is a vector function, $p$ denotes a scalar function, $x \in \mathbb{R}^{n}$ ) describes the dynamics of an incompressible viscous medium with the coefficient of viscosity $\nu$, the velocity vector $Y$ of hydraulic flow and the convective component defined by the term $\sum_{i=1}^{n} Y_{i} \frac{\partial Y}{\partial x_{i}}$ in expression (1). In many cases (first of all, in laminar processes), the convective component is absent, equation (1) becomes linear and system (1), (2) is called the linearized Navier-Stokes system.

The papers [2-5] studied on systematic basis different optimal control problems for evolutionary equations with distributed parameters on a geometrical graph (net). The present work is a natural continuation of the cited research towards increasing the dimension of the spatial variable $x\left(x \in \mathbb{R}^{n}, n \geqslant 2\right)$ and also the dimension of the functions that describe the system state. Note that the simpler case without the convective effect (i. e., the laminar flow of an incompressible viscous medium) is considered below. We touch a rather wide range of optimal control issues for distributed parameter systems on netlike domains, namely, the unique solvability of the corresponding initial boundary value problem and the analysis of optimal control problems that are commonly encountered in applications (distributed and starting control). Sufficient attention is paid to the design of optimal control actions.

2. Necessary notations, concepts and definitions. Consider an open bounded domain $\Im$ of the Euclidean space $\mathbb{R}^{n}$ that has a netlike structure $[3,4]$, i. e., $\Im=$ $\left(\bigcup_{k} \Im_{k}\right) \bigcup\left(\bigcup_{l} S_{l}\right)$, where $S_{l}$ is a surface that separates adjacent domains $\Im_{k}, \partial \Im$ indicates the boundary of $\Im$ (initially, the smoothness of $\partial \Im$ is not important). The locus of conjugation of the adjacent domains $\Im_{k}$ will be called the node locus and further denoted by $\xi$; it represents the union of surfaces $S_{l}(\xi)$ whose number coincides with the number of conjugated domains, that is, $\xi=\bigcup_{l} S_{l}(\xi)$. Throughout the paper, we use measurable 
functions and the Lebesgue integral. The latter over the domain $\Im$ is comprehended as the sum of the integrals over the domains $\Im_{k}$, i. e., $\int_{\Im} f d x=\sum_{k} \int_{\Im_{k}} f d x$.

For a vector function $Y(x, t)=\left\{y_{1}(x, t), y_{2}(x, t), \ldots, y_{n}(x, t)\right\}\left(x=\left\{x_{1}, x_{2}, \ldots, x_{n}\right\}\right)$ defined in a domain $\Im_{T}=\Im \times(0, T)(T<\infty)$, consider the linearized Navier-Stokes system

$$
\begin{gathered}
\frac{\partial Y}{\partial t}-\nu \triangle Y+\nabla p=f, \\
\operatorname{div} Y=0,
\end{gathered}
$$

with the relationships

$$
\begin{gathered}
\left.Y\right|_{S_{l}^{-}(\xi)}=\left.Y\right|_{S_{l}^{+}(\xi)}, \\
\left.\sum_{l} \frac{\partial Y}{\partial n_{l}^{-}}\right|_{S_{l}^{-}(\xi)}+\left.\sum_{l} \frac{\partial Y}{\partial n_{l}^{+}}\right|_{S_{l}^{+}(\xi)}=0
\end{gathered}
$$

holding for each node locus $\xi$ (which are known in the literature as the conjugation conditions, see [3-5]); here $S_{l}^{-}(\xi)$ and $S_{l}^{+}(\xi)$ mean the unilateral surfaces for $S_{l}(\xi)$ defined by the direction of the normals $n_{l}^{-}$and $n_{l}^{+}$to the surfaces $S_{l}^{-}(\xi)$ and $S_{l}^{+}(\xi)$, respectively. Supplementing (3)-(6) with the initial conditions

$$
Y(x, 0)=Y_{0}(x), \quad x \in \Im,
$$

at time $t=0$ and the boundary conditions

$$
\left.Y\right|_{\partial \Im}=0
$$

on the boundary of the continuous medium volume, we obtain the initial boundary value problem $(3)-(8)$ to find the functions $Y(x, t)$ and $p(x, t)(p(x, t)$ is a scalar function) in the closed domain of $\Im_{T}\left(\bar{\Im}_{T}=(\Im \cup \partial \Im) \times[0, T]\right)$.

In the applied problems of hydrodynamics, the netlike domain $\Im$ is actually a hydrosystem caused by a pressure $p$ (with a pressure gradient $\nabla p=\operatorname{grad} p$ ) that distributes the flows of a fluid (a multiphase medium); the function $Y(x, t)$ describes the velocity vector of the hydraulic flow in the domain $\Im_{T}$; relationships $(3),(4)$ reflect the dynamics of an incompressible fluid with the coefficient of viscosity $\nu>0$ in the domain $\bigcup_{k} \Im_{k} \times(0, T)$; the balance equalities (5), (6) define the fluid flow conditions in the node loci of the hydrosystem $\Im$; finally, $f(x, t)$ denotes the density of external forces. The transfer process of the multistage medium is isothermal.

Let us define a weak solution of the initial boundary value problem (3)-(8). To this end, introduce necessary spaces and present preliminary considerations.

Designate by $L_{2}(\Im)^{n}$ the space of measurable functions (classes) $\mu=\left\{\mu_{1}, \mu_{2}, \ldots, \mu_{n}\right\}$ that are square summable over the domain $\Im$. For $\mu, \rho \in L_{2}(\Im)^{n}$, the scalar product is defined by

$$
(\mu, \rho)=\sum_{i=1}^{n} \int_{\Im} \mu_{i}(x) \rho_{i}(x) d x,
$$

where $\|\mu\|=(\mu, \mu)^{1 / 2}$. Assume that $D(\Im)^{n}$ forms the space of functions that are infinitely differentiable in the domain $\Im$ and have compact supports in $\Im$. Let $\mathfrak{D}(\Im)^{n}=\{\phi: \phi \in$ $\left.D(\Im)^{n}, \operatorname{div} \phi=0\right\}$ and denote by $\mathfrak{D}^{\prime}(\Im)^{n}$ the conjugate space for $\mathfrak{D}(\Im)^{n}$ (here and in the sequel, the symbol ' indicates conjugate spaces). Define the space $\mathcal{H}(\Im)$ as the closure of $\mathfrak{D}(\Im)^{n}$ in the norm $L_{2}(\Im)^{n}$ with the scalar product $(9),\|\mu\|_{\mathcal{H}(\Im)}=(\mu, \mu)^{1 / 2}$ and $\mathcal{H}(\Im)=$ $\mathcal{H}(\Im)^{\prime}$. 
Consider a space $\mathcal{H}^{1}(\Im)$ consisting of the elements $\mu \in L_{2}(\Im)^{n}$ that have the generalized derivative $\frac{\partial \mu}{\partial x} \in L_{2}(\Im)^{n}\left(\frac{\partial \mu}{\partial x_{i}} \in L_{2}(\Im), i=\overline{1, n}\right)$. The space $\mathcal{H}^{1}(\Im)$ is equipped with the norm $\|\mu\|_{\mathcal{H}^{1}(\Im)}=\left(\|\mu\|_{L_{2}(\Im)}^{2}+\left\|\frac{\partial \mu}{\partial x}\right\|_{L_{2}(\Im)}^{2}\right)^{1 / 2}$ and represents a Hilbert space with the scalar product $(\mu, \rho)_{\mathcal{H}^{1}(\Im)}=(\mu, \rho)+\left(\frac{\partial \mu}{\partial x}, \frac{\partial \rho}{\partial x}\right)$.

Next, define a space $V_{0}^{1}(\Im)$ as the closure in the norm $\mathcal{H}^{1}(\Im)$ for the set of elements $\mu \in \mathfrak{D}(\Im)^{n}$ satisfying the conjugation conditions $\left.\sum_{l} \frac{\partial \mu}{\partial n_{l}^{-}(\xi)}\right|_{S_{l}^{-}(\xi)}+\left.\sum_{l} \frac{\partial \mu}{\partial n_{l}^{+}}\right|_{S_{l}^{+}(\xi)}=0$. In other words, $V_{0}^{1}(\Im)$ is the subspace of functions from $\mathcal{H}^{1}(\Im)$ that "satisfy the conjugation conditions" in all node loci $\xi$ of the domain $\Im$ and "vanish" on $\partial \Im$. Note that the space $V_{0}^{1}(\Im)$ can be equivalently defined as the closure in the norm $\mathcal{H}^{1}(\Im)$ for the set of elements $\mu \in \mathfrak{D}_{\circ}(\Im)^{n} \subset \mathfrak{D}(\Im)^{n}$ (in contrast to the elements of the set $\mathfrak{D}(\Im)^{n}$, the ones of $\mathfrak{D} \circ(\Im)^{n}$ satisfy the stated conjugation conditions).

Consider the bilinear form

$$
\rho(u, v)=\sum_{i, j=1}^{n} \int_{\Im} \frac{\partial u_{j}}{\partial x_{i}} \frac{\partial v_{j}}{\partial x_{i}} d x,
$$

in functions $u, v, \omega$ such that the integrals in the above representation are convergent.

Lemma. The bilinear form (10) is continuous on $V_{0}^{1}(\Im) \times V_{0}^{1}(\Im)$.

P r o o f. Applying the Cauchy-Bunyakowsky-Schwartz inequality to the functions $\frac{\partial u_{j}}{\partial x_{i}}$ and $\frac{\partial v_{j}}{\partial x_{i}}$ in the right-hand side of form (10) yields

$$
\left|\int_{\Im} \frac{\partial u_{j}}{\partial x_{i}} \frac{\partial v_{j}}{\partial x_{i}} d x\right| \leqslant \sqrt{\int_{\Im}\left(\frac{\partial u_{j}}{\partial x_{i}}\right)^{2} d x} \sqrt{\int_{\Im}\left(\frac{\partial v_{j}}{\partial x_{i}}\right)^{2} d x} \leqslant\left\|u_{j}\right\|_{V_{0}^{1}(\Im)}\left\|v_{j}\right\|_{V_{0}^{1}(\Im)} .
$$

And the statement of this lemma follows from inequality (11).

Introduce the spaces of functions $u(x, t)$ of the variables $x, t \in \Im_{T}$ and consider $u$ as a function of $t$ taking values in the space of functions of $x$. Particularly, if $V$ represents a Hilbert space, then denote by $L_{2}(0, T ; V)$ the space of functions (classes) $u:(0, T) \rightarrow V$ that are measurable, take values from $V$ and

$$
\|u\|_{L_{2}(0, T ; V)}=\left(\int_{0}^{T}\|u(t)\|_{V}^{2} d t\right)^{1 / 2}<\infty .
$$

Note that the equality $L_{2}\left(\Im_{T}\right)^{n}=L_{2}\left(0, T ; L_{2}(\Im)^{n}\right)$ holds obviously.

Next, introduce the following spaces: $W^{1,0}\left(\Im_{T}\right)$ as the space of functions $u(x, t) \in$ $L_{2}\left(\Im_{T}\right)^{n}$ that have the generalized first derivative with respect to $x$ belonging to $L_{2}\left(\Im_{T}\right)^{n}$, with the norm in $W^{1,0}\left(\Im_{T}\right)$ defined by

$$
\|u\|_{W^{1,0}\left(\Im_{T}\right)}=\left(\|u\|_{L_{2}\left(\Im_{T}\right)^{n}}^{2}+\left\|\frac{\partial u}{\partial x}\right\|_{L_{2}\left(\Im_{T}\right)^{n}}^{2}\right)^{1 / 2} ;
$$

$W^{1}\left(\Im_{T}\right)$ as the space of functions from $L_{2}\left(\Im_{T}\right)^{n}$ that have the generalized first derivatives also belonging to $L_{2}\left(\Im_{T}\right)^{n}$, with the norm defined by

$$
\|u\|_{W^{1}\left(\Im_{T}\right)}=\left(\|u\|_{L_{2}\left(\Im_{T}\right)^{n}}^{2}+\left\|\frac{\partial u}{\partial t}\right\|_{L_{2}\left(\Im_{T}\right)^{n}}^{2}+\left\|\frac{\partial u}{\partial x}\right\|_{L_{2}\left(\Im_{T}\right)^{n}}^{2}\right)^{1 / 2} .
$$

Using the properties of the traces of elements from $W^{1}\left(\Im_{T}\right)$ on each section of $\Im_{T}$ by the plane $t=t_{0}\left(t_{0} \in[0, T]\right)$ as elements of $L_{2}(\Im)^{n}$ that are continuous in $t$ in the norm $L_{2}(\Im)^{n}$ (see [6, p. 70]), define $\Omega_{0}\left(\Im_{T}\right)$ as the set of functions $u(x, t) \in W^{1}\left(\Im_{T}\right)$ belonging to the class $V_{0}^{1}(\Im)$ for fixed $t \in[0, T]$. Denote by $W_{0}^{1}\left(\Im_{T}\right)$ the closure of the set $\Omega_{0}\left(\Im_{T}\right)$ in the norm $W^{1}\left(\Im_{T}\right)$. 
Next, let $\widehat{\Omega}_{0}\left(\Im_{T}\right)$ be the set of all functions $u(x, t) \in W^{1,0}\left(\Im_{T}\right)$ that

a) have the finite norm

$$
\|u\|_{2, \Im_{T}}=\max _{0 \leqslant t \leqslant T}\|u(\cdot, t)\|_{L_{2}(\Im)^{n}}+\left\|\frac{\partial u}{\partial x}\right\|_{L_{2}\left(\Im_{T}\right)^{n}}
$$

b) have the trace defined on the sections of the domain $\Im_{T}$ by the plane $t=t_{0}$ $\left(t_{0} \in[0, T]\right)$ as a function of the class $V_{0}^{1}(\Im)$, i. e., for each element $u \in \widehat{\Omega}_{0}\left(\Im_{T}\right)$ and fixed $t \in[0, T]$ there exists a sequence $\left\{u_{n}\right\}$ of functions $u_{n}(x, t) \in V_{0}^{1}(\Im)$ converging to this trace in the norm $\mathcal{H}^{1}(\Im)$;

c) are continuous in $t$ in the norm $\mathcal{H}^{1}(\Im)$ on $[0, T]$, i. e., for any $t \in[0, T], \| u(\cdot, t+$ $\Delta t)-u(\cdot, t) \|_{\mathcal{H}^{1}(\Im)} \rightarrow 0$ as $\Delta t \rightarrow 0$ uniformly on the interval $[0, T]$.

Designate by $V_{0}^{1,0}\left(\Im_{T}\right)$ the closure of the set $\widehat{\Omega}_{0}\left(\Im_{T}\right)$ in norm (12); in addition, $W_{0}^{1,0}\left(\Im_{T}\right)$ is the closure of the set $\widehat{\Omega}_{0}\left(\Im_{T}\right)$ in the norm $W^{1,0}\left(\Im_{T}\right)$ whose elements possess property (b) only. Clearly, $V_{0}^{1,0}\left(\Im_{T}\right) \subset W_{0}^{1,0}\left(\Im_{T}\right) \subset W^{1,0}\left(\Im_{T}\right)$. The space $V_{0}^{1,0}\left(\Im_{T}\right)$ is used below to prove the solvability of problem $(3)-(8)$, whereas the spaces $W_{0}^{1,0}\left(\Im_{T}\right)$, $W^{1,0}\left(\Im_{T}\right)$, and $W_{0}^{1}\left(\Im_{T}\right)$ play auxiliary roles.

Remark 1. If $Y \in V_{0}^{1,0}\left(\Im_{T}\right)$, then $Y=0$ on $\partial \Im$, i. e., relationships (5), (6), and (8) should be treated as the conditions of belonging of $Y$ to the space $V_{0}^{1,0}\left(\Im_{T}\right)$. Equality (7) is considered almost everywhere on $\Im$.

Remark 2. The statement of Lemma remains in force for the functions that are defined in the domain $\Im_{\tau}=\Im \times(0, \tau)$ and have traces for any $t \in(0, \tau)$, where $\tau$ takes any fixed value within the interval $[0, T]$. The proof of this result is exactly the same.

Remark 2 naturally leads to the following definition of the solution of problem (3)-(8), where the initial data (i. e., the functions $f$ and $Y_{0}$ ) satisfy the conditions

$$
f(x, t) \in L_{2,1}\left(\Im_{T}\right), \quad Y_{0}(x) \in \mathcal{H}(\Im) .
$$

Here $L_{2,1}\left(\Im_{T}\right)$ is a space whose elements belong to $L_{1}\left(\Im_{T}\right)$ and have the finite norm $\|f\|_{L_{2,1}\left(\Im_{T}\right)}=\int_{0}^{T}\left(\int_{\Im} f^{2} d x\right)^{1 / 2} d t, \quad L_{2}\left(\Im_{T}\right) \subset L_{2,1}\left(\Im_{T}\right)$.

Definition. A weak solution of the initial boundary value problem (3)-(8) is a pair $\{Y, p\}$ as follows. The function $Y(x, t) \in V_{0}^{1,0}\left(\Im_{T}\right)$ satisfies the integral identity

$$
\begin{gathered}
(Y(x, t), \eta(x, t))-\int_{\Im_{t}} Y(x, \tau) \frac{\partial \eta(x, \tau)}{\partial \tau} d x d \tau+\nu \int_{0}^{t} \rho(Y, \eta) d \tau= \\
=\left(Y_{0}(x), \eta(x, 0)\right)+\int_{\Im_{t}} f(x, \tau) \eta(x, \tau) d x d \tau
\end{gathered}
$$

for any $t \in[0, T]$ and any $\eta(x, t) \in W_{0}^{1}\left(\Im_{T}\right)$, while the function $p(x, t)$ belongs to the class $\mathfrak{D}^{\prime}\left(\Im_{T}\right)^{n}$. Here $\mathfrak{D}^{\prime}\left(\Im_{T}\right)^{n}$ is the conjugate space for the space $\mathfrak{D}\left(\Im_{T}\right)^{n}$, the elements of $\mathfrak{D}\left(\Im_{T}\right)^{n}$ are infinitely differentiable in $\Im_{T}$ functions with a compact support from $\Im_{T}$ ( see the analogous spaces $\mathfrak{D}(\Im)^{n}$ and $\left.\mathfrak{D}^{\prime}(\Im)^{n}\right)$.

Remark 3. Despite the apparent rigor, the above definition of a solution of problem (3)-(8) (i. e., a pair $\{Y, p\}$ ) has the explicit ambiguity caused by the variational statement (14) of this problem "eliminating" the function $p(x, t)$. Notably, there is no information concerning the function $p(x, t)$ except for relationship (14), and hence it suffices to find an appropriate function $p(x, t)$ within the class, i. e., $p(x, t) \in \mathfrak{D}^{\prime}\left(\Im_{T}\right)^{n}$. In terms of applications, this is an acceptable condition that guarantees the nonzero fluid dynamics in the domain $\Im_{T}$ (as a matter of fact, in many applications $p(x, t)$ is an a priori 
given function). According to the aforesaid, in the sequel we consider the function $Y(x, t)$ as a "solution" of problem (3)-(8); the existence of the function $p(x, t)$ and its belonging to the class $\mathfrak{D}^{\prime}\left(\Im_{T}\right)^{n}$ directly follow from the existence of $Y(x, t)$ within the class $V_{0}^{1,0}\left(\Im_{T}\right)$.

The forthcoming sections are dedicated to the unique weak solvability of the initial boundary value problem (3)-(8) and the optimal control problem of system (3)-(6). Some considerations are similar to the ones presented in [1, p. 77] and [3-5].

3. Unique weak solvability of problem (3)-(8). The idea to prove the existence of a unique weak solution of problem (3)-(8) remains the same as in [3-5] for similar distributed parameter problems on a geometrical graph (network), but there is an essential difference that forms the main obstacle. In particular, the domain of variation of the spatial variable in problem (3)-(8) is the bounded domain $\Im$ of the Euclidean space $\mathbb{R}^{n}$, i. e., the spatial variable and the function $Y(x, t)$ are both vectors. This feature causes additional technical difficulties, mostly affecting the structure and properties of the spaces that are selected to describe the weak solutions of problem $(3)-(8)$.

Prior to proving the existence of a weak solution of the initial boundary value problem (3)-(8), in the domain $\Im$ consider the spectral problem

$$
-\nu \triangle U=\lambda U,\left.\quad U\right|_{\partial \Im}=0,
$$

by analogy to its counterpart on a graph [7]. This problem is to find the set of numbers $\lambda$ each associated with at least one nontrivial solution $U(x) \in V_{0}^{1}(\Im)$ satisfying the identity

$$
\nu((U, \eta))=\lambda(U, \eta)
$$

for any function $\eta(x) \in V_{0}^{1}(\Im)$; here $((\cdot, \cdot))$ denotes the scalar product of the form

$$
((U, \eta))=\sum_{i=1}^{n}\left(\frac{\partial U}{\partial x_{i}}, \frac{\partial \eta}{\partial x_{i}}\right)_{L_{2}(\Im)^{n}} .
$$

This means that $U(x)$ is a generalized eigenfunction from the class $V_{0}^{1}(\Im)$ and $\lambda$ is the corresponding eigenvalue. Then the following properties of the eigenvalues and generalized eigenfunctions still take place, by analogy with the ones presented in the paper [2].

1. The eigenvalues are real and have finite multiplicities, and they can be indexed in the ascending order of their magnitudes taking into account multiplicities, $i . e .,\left\{\lambda_{i}\right\}_{i \geqslant 1}$; the generalized eigenfunctions are indexed accordingly, $\left\{U_{i}(x)\right\}_{i \geqslant 1}$.

2. The system of the generalized eigenfunctions $\left\{U_{i}(x)\right\}_{i \geqslant 1}$ forms an orthogonal basis in the spaces $V_{0}^{1}(\Im)$ and $L_{2}(\Im)^{n}$.

Remark 4. These properties remain in force for the spectral problem, where the boundary condition $\left.U\right|_{\partial \Im}=0$ is replaced by the more general one of the form

$$
\frac{\partial U}{\partial n}+\left.\sigma U\right|_{\partial \Im}=0
$$

(each domain $\partial \Im_{l} \cap \partial \Im$ has a specific constant $\sigma$, and $\frac{\partial U}{\partial n}$ designates the derivative along the inner normal inside the domain $\Im$ ). In this case, the generalized eigenfunction belongs to the space $V^{1}(\Im)$ (the definitions of the spaces $V^{1}(\Im)$ and $V_{0}^{1}(\Im)$ differ in the boundary conditions, $\left.V\right|_{\partial \Im}=0$ and the above-mentioned general boundary condition, used in the description of the set $\Omega$ ) and also satisfies the identity

$$
\nu((U, \eta))+\sigma(U, \eta)_{\partial \Im}=\lambda(U, \eta)
$$

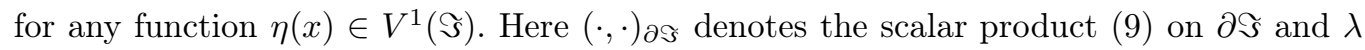
is an eigenvalue. 
Theorem 1. There exists at least one weak solution of the initial boundary value problem (3)-(8) for arbitrary finite $T>0$.

$\mathrm{P}$ r o o f. Use the system of the eigenfunctions $\left\{U_{i}(x)\right\}_{i \geqslant 1}$ as a basis to represent the approximate solution $Y_{m}(x, t)$ in the form

$$
Y_{m}(x, t)=\sum_{i=1}^{m} g_{i m}(t) U_{i}(x)
$$

(the scalar functions $g_{i m}(t)$ are absolutely continuous on $[0, T]$ ) that satisfies the system

$$
\begin{gathered}
\left(\frac{\partial Y_{m}}{\partial t}, U_{i}\right)+\nu \rho\left(Y_{m}, U_{i}\right)=\left(f, U_{i}\right), \quad i=\overline{1, m}, \quad t \in[0, T], \\
Y_{m}(x, 0)=Y_{0 m}(x),
\end{gathered}
$$

where $Y_{0 m}(x)=\sum_{i=1}^{m} g_{i m}^{0} U_{i}(x)\left(g_{i m}^{0}=g_{i m}(0)\right), Y_{0 m}(x) \rightarrow Y_{0}(x)$ in the norm $\mathcal{H}(\Im)$.

System $(15),(16)$ is a system of differential equations in the functions $g_{i m}(t)$, which allows to find $Y_{m}$ for any $t \in[0, T]$. Let us demonstrate this by obtaining the a priori estimates of the norms of $Y_{m}$ in $V_{0}^{1,0}\left(\varsigma_{T}\right)$.

Multiplication of (15) by $g_{i m}(t)$ and summation over $i=\overline{1, m}$ yield

$$
\frac{1}{2} \frac{\partial}{\partial t}\left\|Y_{m}\right\|_{L_{2}(\Im)^{n}}^{2}+\nu \rho\left(Y_{m}, Y_{m}\right)=\left(f, Y_{m}\right) .
$$

In expression (17), the left-hand side is $\frac{1}{2} \frac{\partial}{\partial t}\left\|Y_{m}\right\|_{L_{2}(\Im)^{n}}^{2}+\nu\left\|\left(Y_{m}\right)_{x}\right\|_{L_{2}(\Im)^{n}}^{2}$ and the righthand side obeys the estimate $\left(f, Y_{m}\right) \leqslant\|f\|_{L_{2}(\Im)^{n}}\left\|Y_{m}\right\|_{L_{2}(\Im)^{n}}$. In combination with (17), it follows that

$$
\frac{1}{2} \frac{\partial}{\partial t}\left\|Y_{m}\right\|_{L_{2}(\Im)^{n}}^{2}+\nu\left\|\left(Y_{m}\right)_{x}\right\|_{L_{2}(\Im)^{n}}^{2} \leqslant\|f\|_{L_{2}(\Im)^{n}}\left\|Y_{m}\right\|_{L_{2}(\Im)^{n}} .
$$

And integration over $t$ between 0 and $t$ gives the inequality

$$
\begin{gathered}
\frac{1}{2}\left\|Y_{m}\right\|_{L_{2}(\Im)^{n}}^{2}+\nu \int_{0}^{t}\left\|\left(Y_{m}\right)_{x}\right\|_{L_{2}\left(\Im_{t}\right)^{n}}^{2} d \tau \leqslant \\
\leqslant \frac{1}{2}\left\|Y_{m}(\cdot, 0)\right\|_{L_{2}(\Im)^{n}}^{2}+\|f\|_{L_{2,1}\left(\Im_{t}\right)}^{2} \max _{\tau \in[0, t]}\left\|Y_{m}(\cdot, \tau)\right\|_{L_{2}(\Im)^{n}}
\end{gathered}
$$

for arbitrary $t \in[0, T]$.

Introduce the notation $z(t)=\max _{\tau \in[0, t]}\left\|Y_{m}(\cdot, \tau)\right\|_{L_{2}(\Im)}$ and multiply the both sides of inequality (18) by 2 to get

$$
z^{2}(t)+2 \nu\left\|\left(Y_{m}\right)_{x}\right\|_{L_{2}\left(\Im_{t}\right)}^{2} \leqslant\left\|Y_{m}(\cdot, 0)\right\|_{L_{2}(\Im)^{n}} z(t)+2\|f\|_{L_{2,1}\left(\Im_{t}\right)}^{2} z(t)
$$

as long as $\left\|Y_{m}(\cdot, 0)\right\|_{L_{2}(\Im)^{n}}^{2} \leqslant z(t)$. Then

$$
\begin{gathered}
z^{2}(t) \leqslant J(t), \\
\left\|\left(Y_{m}\right)_{x}\right\|_{L_{2}\left(\Im_{t}\right)^{n}}^{2} \leqslant \frac{1}{2 \nu} J(t),
\end{gathered}
$$

with $J(t)=\left\|Y_{m}(\cdot, 0)\right\|_{L_{2}(\Im)^{n}} z(t)+2\|f\|_{L_{2,1}\left(\Im_{t}\right)^{n}}^{2} z(t)$. The last two inequalities lead to the estimate

$$
\begin{aligned}
& \left\|Y_{m}\right\|_{2, \Im_{t}}=z(t)+\left\|\left(Y_{m}\right)_{x}\right\|_{L_{2}\left(\Im_{t}\right)^{n}} \leqslant\left(1+\frac{1}{\sqrt{2 \nu}}\right) J^{1 / 2}(t) \leqslant \\
& \leqslant\left(1+\frac{1}{\sqrt{2 \nu}}\right)\left(\left\|Y_{m}(\cdot, 0)\right\|_{L_{2}(\Im)^{n}}+2\|f\|_{L_{2,1}\left(\Im_{t}\right)}\right)^{1 / 2}\left\|Y_{m}\right\|_{2, \Im_{t}}^{1 / 2}
\end{aligned}
$$

or, for any $t \in[0, T]$,

$$
\left\|Y_{m}\right\|_{2, \Im_{t}} \leqslant\left(1+\frac{1}{\sqrt{2 \nu}}\right)^{2}\left(\left\|Y_{m}(\cdot, 0)\right\|_{L_{2}(\Im)^{n}}+2\|f\|_{L_{2,1}\left(\Im_{t}\right)}\right) .
$$


Taking into consideration formula (19), the expansion $Y_{0 m}(x)=\sum_{i=1}^{m} g_{i m}^{0} U_{i}(x)$, as the convergence $Y_{0 m}(x) \rightarrow Y_{0}(x)$ in the norm $\mathcal{H}(\Im)$, we have $\left\|Y_{m}(\cdot, 0)\right\|_{L_{2}(\Im)^{n}} \leqslant c\left\|Y_{0}\right\|_{L_{2}(\Im)^{n}}$ (where $c>0$ is a constant independent of $m$ ) and, using (19), the estimate

$$
\begin{aligned}
\left\|Y_{m}\right\|_{2, \Im_{t}} & \leqslant\left(1+\frac{1}{\sqrt{2 \nu}}\right)^{2}\left(c\left\|Y_{0}\right\|_{L_{2}(\Im)^{n}}+2\|f\|_{L_{2,1}\left(\Im_{t}\right)}\right) \leqslant \\
& \leqslant C^{*}\left(\left\|Y_{0}\right\|_{L_{2}(\Im)^{n}}+2\|f\|_{L_{2,1}\left(\Im_{t}\right)}\right),
\end{aligned}
$$

where $C^{*}>0$ is a constant independent of $m$. The resulting estimate (20) plays a dual role:

1) for any index $m$, the norms of the approximate solutions $Y_{m}(x, t)$ and their generalized derivatives $\frac{\partial Y_{m}(x, t)}{\partial x}$ in the space $\mathcal{H}(\Im)$ are bounded by a constant $C$ that is independent of $m$, i. e., $\left\|Y_{m}\right\|_{L_{2}(\Im)^{n}} \leqslant C, \quad\left\|\frac{\partial Y_{m}(\cdot, t)}{\partial x}\right\|_{L_{2}(\Im)^{n}} \leqslant C$;

2) for any index $m$, the norms of the approximate solutions $Y_{m}(x, t)$ are estimated by the norms of the initial data $Y_{0}(x)$ and $f(x, t)$ of the initial boundary value problem (3)-(8).

With this in mind, employ the following well-known result for a sequence $\left\{Y_{m}\right\}_{m \geqslant 1}$ with the collectively bounded norms of elements, see [7, p. 31]. In a sequence $\left\{Y_{m}\right\}_{m \geqslant 1}$, it is possible to separate a subsequence $\left\{Y_{m_{k}}\right\}_{k \geqslant 1}$ that weakly converges in norm (12) to a certain element $Y \in V_{0}^{1,0}\left(\Im_{T}\right)$ (in fact, $\left\{Y_{m_{k}}\right\}_{k \geqslant 1}$ weakly converges to $Y$ in the norm $L_{2}\left(\Im_{T}\right)^{n}$ together with $\left.\frac{\partial Y_{m_{k}}}{\partial x}\right)$. Show that the element $Y(x, t)$ is a solution of problem $(3)-(8)$.

Multiply expression (15) by an absolutely continuous on $[0, T]$ function $d_{i}(t)$, perform summation over $i=\overline{1, m}$ and integrate the result over $t$ between 0 and $t$ :

$$
\begin{gathered}
\left(Y_{m}(x, t), \Phi_{m}(x, t)\right)-\int_{\Im_{t}} Y_{m}(x, \tau) \frac{\partial \Phi_{m}(x, \tau)}{\partial \tau} d x d \tau+\nu \int_{0}^{t} \rho\left(Y_{m}, \Phi_{m}\right) d \tau= \\
=\left(Y_{0}(x), \Phi_{m}(x, 0)\right)+\int_{\Im_{t}} f(x, \tau) \Phi_{m}(x, \tau) d x d \tau
\end{gathered}
$$

where $\Phi_{m}(x, t)=\sum_{i=1}^{m} d_{i}(t) U_{i}(x)$.

Denote by $\Sigma$ the set of all functions $\Phi_{m}(x, t)$ with arbitrary $d_{i}(t)$ possessing the above properties and with arbitrary natural indexes $m$. The set $\Sigma$ is dense in $W_{0}^{1}\left(\Im_{T}\right)$. This follows from the density of the set $\left\{U_{i}(x)\right\}_{i \geqslant 1}$ in $V_{0}^{1}(\Im)$, the continuity of $\Phi_{m}(x, t)$ in $t$ in the norm $\mathcal{H}^{1}(\Im)$ on $[0, T]$, the belonging $\Phi_{m}(x, t) \in V_{0}^{1}(\Im)$ for each fixed $t \in[0, T]$, and the definition of the space $W_{0}^{1}\left(\Im_{T}\right)$.

In (21), fix the function $\Phi_{m}(x, t)=\Phi_{m^{*}}^{*}(x, t) \in \Sigma$, i. e.,

$$
\Phi_{m^{*}}^{*}(x, t)=\sum_{i=1}^{m^{*}} d_{i}^{*}(t) U_{i}(x),
$$

and, starting from index $m_{k} \geqslant m^{*}$, pass to the limit in the above subsequence $\left\{Y_{m_{k}}\right\}_{k \geqslant 1}$, observing that the integral $\int_{0}^{t} \rho\left(Y_{m_{k}}, \Phi_{m^{*}}^{*}\right) d \tau$ converges to $\int_{0}^{t} \rho\left(Y, \Phi_{m^{*}}^{*}\right) d \tau$ by Lemma. Passage to the limit yields formula $(21)$ for the limiting function $Y(x, t)$, and hence for $\eta(x, t)=\Phi_{m^{*}}^{*}(x, t)$ the function $Y(x, t)$ is a weak solution of the initial boundary value problem (3)-(8) from $V_{0}^{1,0}\left(\Im_{T}\right)$ due to the density of the set $\Sigma$ in $W_{0}^{1}\left(\Im_{T}\right)$.

To complete the proof, it remains to argue the existence of a function $p(x, t) \in$ $\mathfrak{D}^{\prime}\left(\Im_{T}\right)^{n}$. Here the line or reasoning is as follows. The obtained function $Y(x, t)$ as a weak solution of problem (3)-(8) satisfies identity (14) for $t=T$; therefore, by choosing 
formally $\frac{\partial Y}{\partial t}-\nu \triangle Y+\sum_{i=1}^{n} Y_{i} \frac{\partial Y}{\partial x_{i}}-f=F$, we have $(F, \eta)=0$ for any element $\eta \in \mathfrak{D}\left(\Im_{T}\right)^{n}$ due to (13) and (14) (recall that $\mathfrak{D}\left(\Im_{T}\right)^{n}$ is dense in $W_{0}^{1}\left(\Im_{T}\right)$, which means the belonging of $F$ to the space $\left.\mathfrak{D}^{\prime}\left(\Im_{T}\right)^{n}\right)$. Moreover, this element has the representation $F=-\operatorname{grad} p$, where the function $p(x, t)$ is a certain element of the space $\mathfrak{D}^{\prime}\left(\Im_{T}\right)^{n}$. The proof of Theorem 1 is finished.

Remark 5. The proof of this theorem contains a deeper statement regarding a weak solution $Y(x, t)$ : the function $Y(x, t)$ has the derivative $\frac{\partial Y(x, t)}{\partial t}$ with respect to the variable $t$ that belongs to the class $L_{2}\left(0, T ; V_{0}^{1}(\Im)\right)$, which follows from the representation of the elements $Y_{m_{k}}(x, t)$ of the subsequence $\left\{Y_{m_{k}}\right\}_{k \geqslant 1}$ for the limiting function $Y(x, t)$.

Now, show that problem (3)-(8) does not have two different solutions from the class $V_{0}^{1,0}\left(\Im_{T}\right)$. Assume that there exist two such solutions, $Y_{1}(x, t)$ and $Y_{2}(x, t)$. Then their difference $Y(x, t)=Y_{1}(x, t)-Y_{2}(x, t)$ is a weak solution of problem $(3)-(8)(f(x, t)=0$, $\left.Y_{0}(x)=0\right)$ from the class $V_{0}^{1,0}\left(\Im_{T}\right)$. The solution $Y(x, t)$ as the limiting element of the sequence $Y_{m}(x, t)$ satisfies the same estimate as $(20)$ with the zero right-hand side (see the proof of Theorem 1). Hence, $Y(x, t)=0$ and the solutions $Y_{1}(x, t)$ and $Y_{2}(x, t)$ coincide. In fact, we have established the following result.

Theorem 2. The initial boundary value problem (3)-(8) has a unique weak solution in the space $V_{0}^{1,0}\left(\Im_{T}\right)$.

Corollary. A weak solution of the initial boundary value problem (3)-(8) continuously depends on the initial data $f(x, t)$ and $Y_{0}(x)$, which appears from the abovementioned estimate (20). Hence, by Theorems 1 and 2, problem (3)-(8) is well-posed in the sense of Hadamard.

4. Optimal control problem. Next, we study two types of optimal control problems that are common in applications, namely, distributed control and starting control (with terminal observations). In the former case, control action appears in the right-hand side of the Navier-Stokes system (i. e., defines the density of external forces); in the latter case, it defines the initial condition of the system at $t=0$. In both cases, the physical problem is to speed up an incompressible viscous multiphase medium to a given vector velocity field by a given (terminal) time $t=T$.

Consider a given Hilbert space $\mathbb{U}$ of control actions $v(x, t)$ and a given space $V_{0}^{1,0}\left(\Im_{T}\right)$ of the states $Y(v)$ of the Navier-Stokes system. Note that the choice of the state space affects the choice of the control space (and vice versa); in this connection, for optimal distributed and starting control problems we believe that $\mathbb{U}$ is a subspace of $L_{2}\left(\Im_{T}\right)^{n}$ and $L_{2}(\Im)^{n}$, respectively. In both cases, system observations are performed on the domain $\Im_{T}$ at terminal time $t=T$ (other types of observations, e. g., boundary ones are also possible). Let $C: L_{2}\left(\Im_{T}\right)^{n} \rightarrow H$ be a linear continuous operator (the observation operator), with $H$ indicating the space of observations (here and in the sequel, $H=L_{2}(\Im)^{n}$ and observations are perfect in the sense of no noises), i. e., $C Y(v)=D Y(v)(x, T)$, where $D: H \rightarrow H$ is a linear bounded operator. On a closed convex set $\mathbb{U}_{\partial}$ of the space $\mathbb{U}$, introduce an objective function $J(v)$ defined by two operators, namely, the transition operator from the control action $v$ to the state $Y(v)$ and the transition operator from the state $Y(v)$ to the observation $C Y(v)$, i. e.,

$$
J(v)=\left\|C Y(v)-z_{0}\right\|_{H}^{2}+(N v, v)_{\mathbb{U}}=\left\|D Y(v)(x, T)-z_{0}\right\|_{H}^{2}+(N v, v)_{\mathbb{U}},
$$

where $z_{0}(x, t) \in L_{2}\left(\Im_{T}\right)^{n}$ is a given observation; $N: \mathbb{U} \rightarrow \mathbb{U}$ denotes a linear continuous Hermitean operator; $(N v, v)_{U} \geqslant \varsigma\|v\|_{U}(\varsigma>0$ specifies a fixed constant). The term 
$(N v, v)_{\cup}$ in the representation of the objective function $J(v)$ guarantees the coercivity of the quadratic component of the objective function $J(v)$ (see [1, p. 13]).

The optimal distributed (or starting) control problem for the Navier-Stokes system is to find $\inf _{v \in \mathbb{U}_{\partial}} J(v)$. An element $u \in \mathbb{U}_{\partial}$ is called an optimal control action of the system if it minimizes the objective function $J(v)$ on the set $\mathbb{U}_{\partial}$.

Distributed control. Consider a given linear bounded operator $B: \mathbb{U} \rightarrow L_{2,1}\left(\Im_{T}\right)$. Then equation (3) takes the form

$$
\frac{\partial Y}{\partial t}-\nu \triangle Y+\nabla p=f+B v
$$

the state $\{Y(v)(x, t), p(v)(x, t)\}$ of system $(23),(4)-(6)$ is defined by a weak solution of the initial boundary value problem $(22),(4)-(8)$. Its well-posedness in the sense of Hadamard follows from the results of Section 3. The state $Y(v)$ has continuous dependence on the control action $v$ (as the mapping $v \rightarrow Y(v)$ is continuous). Therefore, it is possible to use the minimization methods of positive definite quadratic forms defined on a closed convex set of a Hilbert space [1, Ch. I] (in addition, see [8, Ch. I] and [3, 4]). Further analysis is similar to the papers $[3,4]$, which explored the issues of optimal control design for distributed parameter systems on a graph (network).

For system (23), or (4)-(6), define the conjugate state $\omega(v)(x, t) \in W_{0}^{1}\left(\Im_{T}\right)$, $\omega(v)(x, T)=D^{*}\left(D Y(T ; v)-z_{0}\right)\left(D^{*}\right.$ is the conjugate operator for $\left.D\right)$, as the function satisfying the integral identity

$$
-\int_{\Im_{T}} \frac{\partial \omega(v)(x, \tau)}{\partial \tau} \eta(x, \tau) d x d \tau+\nu \int_{0}^{T} \rho(\omega(v), \eta) d \tau=0
$$

for any functions $\eta(x, t) \in W_{0}^{1,0}\left(\Im_{T}\right)$ (as a matter of fact, this is a variational statement that "eliminates" the function $p(x, t)$ and has obvious analogy with (14)). To prove the existence of a unique weak solution $\omega(v)$, it suffices to apply Theorem 1 , where $t$ is replaced by $T-t$.

Under the above conditions, we arrive at the following result, which is similar to Theorem 5 from [3].

Theorem 3. For an element $u(x, t) \in \mathbb{U}_{\partial}$ to be an optimal distributed control action of system (23), (4)-(6), a necessary and sufficient condition is the following relationships:

$$
\begin{gathered}
(Y(u)(x, t), \eta(x, t))-\int_{\Im_{t}} Y(u)(x, \tau) \frac{\partial \eta(x, \tau)}{\partial \tau} d x d \tau+\nu \int_{0}^{t} \rho(Y(u), \eta) d \tau= \\
=\left(Y_{0}(x), \eta(x, 0)\right)+\int_{\Im_{t}}(f(x, \tau)+B u(x, \tau)) \eta(x, \tau) d x d \tau
\end{gathered}
$$

for any $t \in[0, T]$ and any $\eta(x, t) \in W_{0}^{1}\left(\Im_{T}\right)$;

$$
-\int_{\Im_{T}} \frac{\partial \omega(u)(x, \tau)}{\partial \tau} \eta(x, \tau) d x d \tau+\nu \int_{0}^{T} \rho(\omega(u), \eta) d \tau=0
$$

for any $\eta(x, t) \in W_{0}^{1,0}\left(\Im_{T}\right)$;

$$
\int_{\Im_{T}}(\omega(u)(x, t)+N u(x, t))(v(x, t)-u(x, t)) d x d t \geqslant 0
$$

for any $v \in \mathbb{U}_{\partial}$.

Here $Y(u) \in V_{0}^{1,0}\left(\Im_{T}\right), \omega(u) \in W_{0}^{1}\left(\Im_{T}\right)$, and $\omega(u)(x, T)=D^{*}\left[D Y(T ; u)-z_{0}\right], x \in \Im$. 
Remark 6. A version of distributed control is the case of set point control of system (3)-(6), which plays an important role for applications. The right-hand side $f$ in (3) is replaced by $\sum_{j=1}^{m} v_{j}(t) \otimes \delta\left(x-x_{j}\right)$, where $v(t)=\left\{v_{1}(t), v_{2}(t), \ldots, v_{m}(t)\right\} \in L_{2}(0, T)^{m}$ and $x_{j} \in \bigcup_{k} \Im_{k}$. This setting means that point control actions are applied at fixed points of the domain $\Im$.

The state $Y(v)$ is defined in the variational statement as an element of the space $V_{0}^{1,0}\left(\Im_{T}\right)$ that satisfies the integral identity

$$
\begin{gathered}
(Y(v)(x, t), \zeta(x, t))-\int_{\Im_{t}} Y(v)(x, \tau) \frac{\partial \zeta(x, \tau)}{\partial \tau} d x d \tau+\nu \int_{0}^{t} \rho(Y(v), \zeta) d \tau= \\
=\left(Y_{0}(x), \zeta(x, 0)\right)+\int_{\Im_{t}} f(x, \tau) \zeta(x, \tau) d x d \tau+\sum_{j=1}^{m} \int_{0}^{t} v_{j}(\tau) \zeta\left(x_{j}, \tau\right) d \tau
\end{gathered}
$$

for any $t \in[0, T]$ and any $\zeta(x, t) \in W_{0}^{2,1}\left(\Im_{T}\right)$. Here $W_{0}^{2,1}\left(\Im_{T}\right)$ is the closure of the elements of $\widetilde{\mathfrak{D}}\left(\Im_{T}\right)^{n}$ in the norm of the space $H^{2,1}\left(\Im_{T}\right)=\left\{g: g, \frac{\partial g}{\partial x_{j}}, \frac{\partial^{2} g}{\partial x_{i} \partial x_{j}}, \frac{\partial g}{\partial t} \in L_{2}\left(\Im_{T}\right)^{n}\right\}$ (see the properties of $H^{2,1}\left(\Im_{T}\right)$ in [1, p. 126]). If the boundary $\partial \Im_{T}$ of the domain $\Im_{T}$ is sufficiently smooth, then the conjugate state $\omega(v)$ represents an element of the space $W_{0}^{2,1}\left(\Im_{T}\right)$ such that $\omega(v)(x, T)=0$.

Starting control. Consider another example of control of system (3)-(6), which arises in the course of analyzing the transfer dynamics of multiphase media. A control action $v(x) \in \mathbb{U}$ is applied at the initial time, defining the initial condition $(7)\left(Y_{0}(x)=\right.$ $v(x))$, i. e.,

$$
Y(x, 0)=v(x), \quad x \in \Im .
$$

The state $\{Y(v)(x, t), p(v)(x, t)\}$ of system $(3)-(6)$ is defined by a weak solution $Y(v)(x, t)$ of the initial boundary value problem $(3)-(6),(8),(24)$ (in the integral identity (14), the function $Y_{0}(x)$ is replaced by $\left.v(x)\right)$ and by an arbitrary function $p(x, t)$ belonging to the class $\mathfrak{D}^{\prime}\left(\Im_{T}\right)^{n}$. Like the case of distributed control, the well-posedness of problem (3)-(6), (8), (24) in the sense of Hadamard follows from the results of Section 3. The conjugate state $\omega(v)(x, t)$ of system (3)-(6) is defined by the integral identity (24); in addition, $\omega(v)(x, T)=D^{*}\left(D Y(T ; v)-z_{0}\right), x \in \Im$ (the last equality holds almost everywhere on $\Im)$.

Then we have the following statement, which is analogous to Theorem 7 from [4].

Theorem 4. For an element $u(x) \in \mathbb{U}_{\partial}$ to be an optimal starting control action of system (3)-(6), a necessary and sufficient condition is the relationships:

$$
\begin{gathered}
(Y(u)(x, t), \eta(x, t))-\int_{\Im_{t}} Y(u)(x, \tau) \frac{\partial \eta(x, \tau)}{\partial \tau} d x d \tau+\nu \int_{0}^{t} \rho(Y(u), \eta) d \tau= \\
=(u(x), \eta(x, 0))+\int_{\Im_{t}} f(x, \tau) \eta(x, \tau) d x d \tau
\end{gathered}
$$

for any $t \in[0, T]$ and any $\eta(x, t) \in W_{0}^{1}\left(\Im_{T}\right)$;

$$
-\int_{\Im_{T}} \frac{\partial \omega(u)(x, \tau)}{\partial \tau} \eta(x, \tau) d x d \tau+\nu \int_{0}^{T} \rho(\omega(u), \eta) d \tau=0
$$

for any functions $\eta(x, t) \in W_{0}^{1,0}\left(\Im_{T}\right)$; 


$$
\int_{\Im}(\omega(u)(x, 0)+N u(x))(v(x)-u(x)) d x d t \geqslant 0
$$

for any $v \in \mathbb{U}_{\partial}$.

Here $Y(u) \in V_{0}^{1,0}\left(\Im_{T}\right), \omega(u) \in W_{0}^{1}\left(\Im_{T}\right)$, and $\omega(u)(x, T)=D^{*}\left[D Y(T ; u)-z_{0}\right], x \in \Im$.

5. Synthesis of optimal control (feedback). Consider the optimal control problem without constraints imposed on control actions, i. e., $\mathbb{U}_{\partial}$ coincides with $\mathbb{U}$. Then it is possible to set $v=u \pm \nu$ in relationships (24) and (25). Owing to arbitrary $v \in \mathbb{U}$, these relationships become equalities and hence the optimal distributed and starting control actions satisfy the equalities

$$
\omega(u)(x, t)+N u(x, t)=0, \omega(u)(x, 0)+N u(x)=0,
$$

$u(x, t)=-N^{-1} \omega(u)(x, t)$, and $u(x)=-N^{-1} \omega(u)(x, 0)$. Consequently, the optimal distributed control action is constructed by solving the system of two integral identities (variational relations):

$$
\begin{gathered}
\int_{\Gamma} Y(x, t) \eta(x, t) d x-\int_{\Gamma_{t}} Y(x, t) \frac{\partial \eta(x, t)}{\partial t} d x d t+\ell_{t}(Y, \eta)= \\
=\int_{\Gamma} Y_{0}(x) \eta(x, h) d x+\int_{\Gamma_{t}} f(x, t) \eta(x, t) d x d t-\int_{\partial \Gamma_{t}} N^{-1} \omega(x, t) \eta(x, t) d x d t
\end{gathered}
$$

for any $t \in[0, T]$ and any function $\eta(x, t) \in W_{0}^{1}\left(\Gamma_{T}\right)$;

$$
-\int_{\Im_{T}} \frac{\partial \omega(u)(x, \tau)}{\partial \tau} \zeta(x, \tau) d x d \tau+\nu \int_{0}^{T} \rho(\omega(u), \zeta) d \tau=0
$$

for any functions $\zeta(x, t) \in W_{0}^{1,0}\left(\Im_{T}\right)$. The optimal distributed control action has the form

$$
u(x, t)=-N^{-1} \omega(x, t) .
$$

To find the optimal starting control action, one has to solve the system of integral identities that is similar to (26) and (27), in which

$$
u(x)=-N^{-1} \omega(x, 0) .
$$

Formulas (28) and (29) realize the synthesis of optimal distributed and starting control of systems $(23),(4)-(6)$ and (3)-(6), respectively. More specifically, optimal control actions are defined through conjugate states, which actually implements the feedback principle by the states of each system.

Remark 7. Under the accepted assumptions, it is possible to establish deeper results of the synthesis of optimal control, that generalize the well-known Kalman results for bounded operators.

6. Conclusion. This paper has considered the applied problems of distributed and starting control for the linearized Navier-Stokes system. The optimal control conditions in terms of the conjugate states of systems (23), (4)-(6) and (3)-(6) have been established, as well as some analogues of the well-known Kalman results of the finite-dimensional case (formulas (28) and (29)) have been obtained. The described algorithm is applicable to many optimization problems for differential systems whose states are defined by weak solutions of evolutionary equations on similar networks as in the papers [3-5]. Interestingly, other researchers considered alternative approaches to the stability analysis [9-11] and 
stabilization $[12,13]$ of the solutions to some applications-relevant classes of complex systems, yet with the same treatment of the optimal control existence conditions. Also note that the problem under study may have peculiarities in form of stochastic components [14] or discontinuous nonlinearities [15] in the representation of evolutionary systems (3) and $(22)$.

\section{References}

1. Lions J.-L. Some methods of solving non-linear boundary value problems. Paris, Dunod-GauthierVillars, 1969, 581 p. (Russ. ed.: Lions J.-L. Nekotorye metody resheniya nelineinykh kraevykh zadach. Moscow, Mir Publ., 1972, 587 p.).

2. Provotorov V. V., Gnilitskaya Yu. A. Boundary control of a wave system in the space of generalized solutions on a graph. Vestnik of Saint Petersburg University. Series 10. Applied Mathematics. Computer Science. Control Processes, 2013, iss. 3, pp. 112-120.

3. Provotorov V. V. Optimal control of a parabolic system with distributed parameters on a graph. Vestnik of Saint Petersburg University. Series 10. Applied Mathematics. Computer Science. Control Processes, 2014, iss. 3, pp. 154-163.

4. Podvalny S. L., Provotorov V. V. Starting control of a parabolic system with distributed parameters on a graph. Vestnik of Saint Petersburg University. Series 10. Applied Mathematics. Computer Science. Control Processes, 2015, iss. 3, pp. 126-142.

5. Podvalny S. L., Provotorov V. V. The questions of controllability of a parabolic systems with distributed parameters on the graph. 2015 Intern. Conference "Stability and Control Processes" in memory of V. I. Zubov (SCP), 2015, pp. 117-119.

6. Ladyzhenskaya O. A. Kraevye zadachi matematicheskoi fiziki [Boundary value problems of mathematical physics]. Moscow, Nauka Publ., 1973, 407 p. (In Russian).

7. Volkova A. S., Provotorov V. V. Generalized solutions and generalized eigenfunctions of boundary-value problems on a geometric graph. Russian Mathematics (Proceeding of Higher Educational Institutions), 2014, vol. 58, no. 3, pp. 1-13.

8. Fursikov A.V. Optimal'noe upravlenie raspredelennymi sistemami. Teoriya i prilozheniya [Optimal control of distributed systems. Theory and applications]. Novosibirsk, Nauchnaya kniga Publ., 1999, 352 p. (In Russian).

9. Aleksandrov A. Yu., Zhabko A. P. On the asymptotic stability of solutions of nonlinear systems with delay. Siberian Mathematical Journal, 2012, vol. 53, no. 3, pp. 393-403.

10. Aleksandrov A., Aleksandrova E., Zhabko A. Asymptotic stability conditions for certain classes of mechanical systems with time delay. WSEAS Transactions on Systems and Control, 2014, vol. 9, pp. 388-397.

11. Aleksandrov A. Yu., Zhabko A. P. On stability of the solutions of a class of nonlinear delay systems. Autom. Remote Control, 2006, vol. 67, no. 9, pp. 1355-1365.

12. Veremey E. I., Korchanov V. M. Multiobjective stabilization of the dynamic systems from a certain class. Avtomatics and Telemechanics, 1988, no. 9, pp. 126-137.

13. Veremey E. I., Sotnikova M. V. Plasma stabilization by prediction with stable linear approximation. Vestnik of Saint Petersburg University. Series 10. Applied Mathematics. Computer Science. Control Processes, 2011, iss. 1, pp. 116-133.

14. Karelin V. V. Penalty functions in the control problem of an observation process. Vestnik of Saint Petersburg University. Series 10. Applied Mathematics. Computer Science. Control Processes, 2010, iss. 4, pp. 109-114.

15. Kamachkin A. M., Yevstafyeva V. V. Oscillations in a relay control system at an external disturbance. Control Applications of Optimization 2000. Proceedings of the 11th IFAC Workshop, 2000, vol. 2, pp. 459-462.

For citation: Provotorov V. V., Provotorova E. N. Optimal control of the linearized NavierStokes system in a netlike domain. Vestnik of Saint Petersburg University. Applied Mathematics. Computer Science. Control Processes, 2017, vol. 13, iss. 4, pp. 431-443. https://doi.org/10.21638/11701/ spbu10.2017.409

Статья рекомендована к печати проф. А. П. Жабко.

Статья поступила в редакцию 7 октября 2016 г.

Статья принята к печати 12 октября 2017 г. 